\title{
Síndrome metabólico en pacientes mexicanos con hipotiroidismo
}

\section{Metabolic syndrome in Mexican patients with hypothyroidism}

\author{
Miguel Á. Mendoza-Romoํㅗ. Ma. Raquel Ponce-Muñiz², Gabriela V. Escudero-Lourdes ${ }^{3}$, Juan F. Ortiz-Nesme ${ }^{4}$, \\ Alejandra Medina-Tinoco ${ }^{2 *}$, Karla Fabela-MendozA ${ }^{5}$ y Diana L. Reyes-Barbosa ${ }^{5}$ \\ ${ }^{1}$ Coordinación en Investigación; ${ }^{2}$ Unidad de Medicina Familiar N. $047 ;{ }^{3}$ Coordinación en Educación; ${ }^{4}$ Jefatura de Prestaciones Médicas, \\ Delegación San Luis Potosí; ${ }^{5}$ Unidad de Medicina Familiar N. ${ }^{\circ}$ 45. Instituto Mexicano del Seguro Social, San Luis Potosí, México
}

\section{RESUMEN}

Objetivo: Determinar la prevalencia del síndrome metabólico en pacientes mexicanos con hipotiroidismo. Métodos: Se seleccionó una muestra de 293 pacientes con diagnóstico de hipotiroidismo durante un lapso de seis meses. Se analizó el perfil de lípidos, el perfil tiroideo, la glucosa en ayuno, la presión arterial, el peso, la talla y el perímetro abdominal. Se tomaron los criterios de la Federación Internacional de Diabetes para el diagnóstico de síndrome metabólico. Resultados: Del total de pacientes estudiados, un $12 \%$ (35) fueron hombres y un $88 \%$ (258) mujeres, se encontró el $65 \%$ (190) con síndrome metabólico, el cual fue mayor en aquellos con hipotiroidismo subclínico, $76 \%$ (60), que en hipotiroidismo clínico, $60 \%$ (130). Los componentes más frecuentemente encontrados en los pacientes estudiados fueron niveles bajos de colesterol ligado a lipoproteínas de alta densidad en el $82 \%$ (240) y obesidad central en el $77 \%$ (226). Conclusiones: Existe una mayor presencia de síndrome metabólico en pacientes con hipotiroidismo, sobre todo en aquellos con hipotiroidismo subclínico, por lo que se sugiere una búsqueda de ambas enfermedades para un adecuado manejo y control del riesgo cardiovascular.

Palabras clave: Hipotiroidismo. Síndrome metabólico. Obesidad.

\begin{abstract}
Aim: Determine the frequency of metabolic syndrome in Mexican patients with hypothyroidism. Methods: We selected a sample of 293 patients with diagnosis of hypothyroidism during a period of 6 months. Lipid profile, thyroid profile, fasting glucose, blood pressure, weight, height and abdominal perimeter were analyzed. The International Diabetes Federation criteria for metabolic syndrome were used for diagnosis. Results: From the total of studied patients, 12\% (35) were men and 88\% (258) were women, 190 (65\%) were found to have metabolic syndrome. It was higher in those with subclinical hypothyroidism, in $76 \%(60)$, than in those with clinical hypothyroidism, $60 \%$ (130). The components most frequently found were low levels of HDL cholesterol $82 \%$ (240) and central obesity $77 \%$ (226). Conclusions: There is a greater presence of metabolic syndrome in patients with hypothyroidism, especially in those with subclinical hypothyroidism, so a search for both diseases is suggested for proper management and control of cardiovascular risk.
\end{abstract}

Key words: Hypothyroidism. Metabolic syndrome. Obesity.

\section{Correspondencia:}

*Alejandra Medina-Tinoco

E-mail: medinatinoco64@hotmail.com
Fecha de recepción: 18-10-2019

Fecha de aceptación: 09-05-2020

DOI: 10.24875/RME.20001959
Disponible en internet: 09-02-2021

Rev Mex Endocrinol Metab Nutr. 2021;8:28-32

2462-4144 / @ 2020 Sociedad Mexicana de Nutricion y Endocrinologia, AC. Publicado por Permanyer. Éste es un artículo open access bajo la licencia CC BY-NC-ND (http://creativecommons.org/licenses/by-nc-nd/4.0/). 


\section{INTRODUCCIÓN}

El síndrome metabólico (SM), también conocido como síndrome $X$, es definido por la Organización Mundial de la Salud como una patología caracterizada por obesidad abdominal, resistencia a la insulina, hipertensión y dislipidemia ${ }^{1}$. Este conjunto de alteraciones metabólicas fue descrito por primera vez en $1920^{2}$. En 2009, diversas organizaciones se reunieron para acordar una definición y criterios. En esta reunión se reconoció que el riesgo asociado con una medida en particular de la cintura variará en diferentes poblaciones ${ }^{3}$. La prevalencia de SM varía alrededor del mundo, debido a la edad y etnicidad de las poblaciones estudiadas y los criterios diagnósticos aplicados. La prevalencia más alta registrada a nivel mundial es entre nativos americanos, con cerca del $60 \%$ en mujeres entre 45 y 49 años y $45 \%$ en hombres de entre 45 y 49 años ${ }^{4}$.

El hipotiroidismo es la deficiencia hormonal patológica más común ${ }^{5}$. Para establecer el diagnóstico de hipotiroidismo es necesario conocer los antecedentes del paciente, el cuadro clínico y los valores de laboratorio del perfil tiroideo. En el tercer estudio para la nutrición y salud (NHANES III) se encontró un aumento de prevalencia de hipotiroidismo con niveles de hormona estimulante de la tiroides (TSH) superiores a $4.5 \mathrm{mlU} / \mathrm{l}$, sin embargo, hay estudios donde observaron que los límites normales de TSH varían de acuerdo con la edad y raza, por lo que el límite debe ajustarse según la población estudiada 6 . En el NHANES III, la prevalencia general de hipotiroidismo fue del $4.6 \%$, la cual fue similar en población blanca e hispana ${ }^{7}$. El hipotiroidismo puede causar disminución en el gasto cardiaco, aumento de la resistencia vascular sistémica y aterosclerosis, y su tratamiento podría beneficiar los parámetros de disfunción cardiaca ${ }^{8}$. Diversos estudios han encontrado que variaciones en la función tiroidea, dentro del rango de referencia normal, incluso elevaciones moderadas de TSH pueden aumentar la mortalidad cardiovascular ${ }^{9}$.

Existe una interacción íntima de la hormona tiroidea con todos los componentes del SM en modelos animales y humanos. Mientras que algunos de estos mecanismos no son totalmente conocidos en humanos, está cada vez más claro que el hipertiroidismo y el hipotiroidismo inducen resistencia a la insulina, el sello fisiopatológico del $\mathrm{SM}^{10}$. Varios estudios han encontrado que el grosor de la íntima media carotídea, el cual es un marcador inicial de aterosclerosis, es mayor en pacientes con hipotiroidismo subclínico y SM que en aquellos pacientes que padecen solo una de estas enfermedades ${ }^{11}$.

En un metaanálisis de 2016, Yang y otros investigadores encontraron que en asiáticos el riesgo de padecer SM en pacientes con hipotiroidismo subclínico parecía ser mayor, así como una mayor asociación dependiendo de los criterios usados para diagnosticarlo, encontrando que parecía haber mayor asociación con los criterios de la Federación Internacional de Diabetes $\left(\right.$ IDF) ${ }^{12}$. En el mismo año, Bamashmos y Atef realizaron un estudio en Yemen en el que buscaron la prevalencia del SM y sus componentes en pacientes con hipotiroidismo; encontraron SM en el $37 \%$ de los que tienen hipotiroidismo, siendo más alto en pacientes con hipotiroidismo subclínico (76\%) (se usaron los criterios del Programa Nacional de Educación sobre el Colesterol y el III Panel de Tratamiento del Adulto [NCEP:ATPIII]) ${ }^{13}$. El siguiente año, Jaideep Khare y otros investigadores en la India realizaron el mismo estudio pero en su población, encontrando una prevalencia del 53\% por los criterios del NCEP:ATP III, siendo la obesidad central el criterio más encontrado ${ }^{14}$. Mientras que en 2018, Mumtaz y Muhammed realizaron un estudio similar, buscando prevalencia de SM en pacientes hipotiroideos bajo tratamiento con levotiroxina (con los criterios de la IDF) y encontraron un 52\% de SM en pacientes hipotiroideos, además de una relación positiva entre la dosis de levotiroxina y el perímetro abdominal, índice de masa corporal (IMC) y niveles de colesterol ligado a lipoproteínas de baja densidad ${ }^{15}$. El objetivo este estudio fue determinar la frecuencia de SM en pacientes mexicanos con hipotiroidismo.

\section{MATERIAL Y MÉTODOS}

Se trata de un estudio descriptivo, observacional, transversal, con muestreo no probabilístico por conveniencia de casos consecutivos. En la consulta de 
endocrinología del Hospital General de Zona N. 2 de San Luis Potosí, México. Se cuenta con un total de 4,979 pacientes con diagnóstico de hipotiroidismo registrados al momento de este estudio. Se valoró el tamaño de la muestra utilizando la calculadora de la aplicación Epi Info para Android versión 1.4, utilizando una frecuencia esperada del 54\%, límite de confianza del $5 \%$ y nivel de confianza del 95\%. Para el estudio se incluyeron 293 pacientes durante un lapso de seis meses, de diciembre de 2017 a abril de 2018. Se incluyeron pacientes mayores de 18 años con diagnóstico de hipotiroidismo de cualquier tipo, que aceptaran participar en el estudio y se encontraran en tratamiento con levotiroxina. Se excluyeron los pacientes con mal control bioquímico. Bajo consentimiento informado y registro de protocolo número R-2017-2402-57 del Comité de Ética e Investigación 2402, se tomaron medidas de peso, talla, perímetro abdominal y presión arterial, se tomaron del expediente clínico los datos de perfil de lípidos, perfil tiroideo y glucosa en ayuno.

Con base en que todavía se desconoce un único criterio para una mejor clasificación del SM y la utilización de distintos criterios diagnósticos, lo que a su vez limita la comparación de las frecuencias de ella entre poblaciones, se decidió que la definición de la IDF para SM, que desde 2005 ha tenido como diferenciales la obesidad abdominal como prerrequisito, respeta las diferentes etnias, además de un punto de corte menor para considerar anormalidad glucémica, esto permite la comparación de los resultados del presente trabajo con otros estudios internacionales.

Los criterios para este estudio fueron: obesidad central (perímetro abdominal $>88 \mathrm{~cm}$ en mujeres y $>94 \mathrm{~cm}$ en hombres, medidas recomendadas para población latinoamericana por la Asociación Latinoamericana de Diabetes), presión arterial > 130/85 $\mathrm{mm} \mathrm{Hg}$, glucemia en ayuno $>100 \mathrm{mg} / \mathrm{dl}$, triglicéridos $>150 \mathrm{mg} / \mathrm{dl}$, lipoproteínas de alta densidad $(\mathrm{HDL})<50 \mathrm{mg} / \mathrm{dl}$ en mujeres $\mathrm{y}<40 \mathrm{mg} / \mathrm{dl}$ en hombres, realizando el diagnóstico en la presencia de tres de los cinco componentes.

Se realizaron pruebas estadísticas con medidas de tendencia central y dispersión, porcentajes, medias, desviación estándar y rangos; estadística inferencial con chi cuadrada y correlación de Pearson.
Tabla 1. Características demográficas, antropométricas y de laboratorio en pacientes estudiados

\begin{tabular}{lcccc}
\hline $\mathrm{n}=293$ & Mínimo & Máximo & Media & $\mathrm{DE}$ \\
\hline Edad & 18 & 89 & 56.40 & 13.989 \\
\hline Cintura & 80 & 150 & 110.60 & 21.570 \\
\hline $\mathrm{IMC}$ & 19.0 & 35.5 & 26.679 & 4.3476 \\
\hline TAS & 110 & 150 & 119.28 & 8.266 \\
\hline TAD & 70 & 80 & 78.23 & 3.827 \\
\hline TSH & 1.5 & 5.5 & 3.448 & 1.2291 \\
\hline HDL & 10 & 56 & 38.63 & 11.536 \\
\hline TG & 102 & 1245 & 153.50 & 71.107 \\
\hline GLUC & 73 & 150 & 109.16 & 19.272 \\
\hline
\end{tabular}

DE: desviación estándar; IMC: índice de masa corporal; TAS: tensión arterial sistólica; TAD: tensión arterial diastólica; TSH: hormona estimulante de la tiroides; HDL: lipoproteínas de alta densidad; TG: triglicéridos; GLUC: glucosa en ayuno.

\section{RESULTADOS}

De los pacientes estudiados, el 12\% (35) fueron hombres y el $88 \%$ (258) mujeres. De estos pacientes, el $27 \%$ (79) tenía hipotiroidismo subclínico y el 73\% (214) hipotiroidismo clínico. En la tabla 1 se describen las características de todos los pacientes incluidos. Se encontró que el 65\% (190) de los pacientes tenía SM, siendo los niveles bajos de HDL en el $82 \%$ (240), el componente más frecuentemente encontrado, seguido de obesidad central en el $77 \%$ (226).

Al estudiar a los pacientes por grupo de edad y sexo, se encontró que la frecuencia de SM fue del $63 \%$ (102) en el grupo de 18 a 59 años y del $67 \%$ (88) en los mayores de 60 años. En las mujeres el componente del SM más frecuente fue nivel bajo de HDL (85\%); en los hombres fue obesidad central (74\%). En la tabla 2 se encuentra la distribución de los componentes del SM por sexo y grupo de edad.

En los pacientes con hipotiroidismo subclínico, se encontró SM en el 76\% (60) y en los de hipotiroidismo clínico en el $60 \%$ (130). Se encontró una asociación con chi cuadrada significativa entre el tipo de hipotiroidismo y la presencia de SM ( $p=0.023)$.

Todos los pacientes se encontraban con niveles bioquímicos normales de TSH (1.5-5.5 mlU/l) para el valor de referencia del laboratorio institucional, al 
Tabla 2. Frecuencia de los componentes del síndrome metabólico por grupo de edad y sexo

\begin{tabular}{|c|c|c|c|c|c|c|c|}
\hline & \multirow[b]{2}{*}{$\begin{array}{c}\text { Total } \\
\mathrm{n}=293\end{array}$} & \multicolumn{3}{|c|}{18 a 59 años } & \multicolumn{3}{|c|}{ Mayor de 60 años } \\
\hline & & $\begin{array}{c}\text { Total } \\
\mathrm{n}=161 \\
(55 \%)\end{array}$ & $\begin{array}{c}\text { Hombres } \\
\mathrm{n}=20 \\
(7 \%)\end{array}$ & $\begin{array}{c}\text { Mujeres } \\
\mathrm{n}=141 \\
(48 \%)\end{array}$ & $\begin{array}{c}\text { Total } \\
\mathrm{n}=132 \\
(45 \%)\end{array}$ & $\begin{array}{c}\text { Hombres } \\
\mathrm{n}=15 \\
(5 \%)\end{array}$ & $\begin{array}{c}\text { Mujeres } \\
\mathrm{n}=117 \\
(40 \%)\end{array}$ \\
\hline SM & $190(65 \%)$ & 102 (63\%) & 12 (60\%) & 90 (64\%) & 88 (67\%) & $9(60 \%)$ & 79 (67\%) \\
\hline $\begin{array}{l}\text { Obesidad central } \\
\text { Cintura } \geq 88 \mathrm{~cm} \text { mujeres } \\
\text { Cintura } \geq 94 \mathrm{~cm} \text { hombres }\end{array}$ & $226(77 \%)$ & $120(75 \%)$ & $15(75 \%)$ & 105 (74\%) & 106 (80\%) & $11(73 \%)$ & 95 (81\%) \\
\hline $\begin{array}{l}\text { Tensión arterial } \\
\geq 130 / 85 \mathrm{mmHg}\end{array}$ & $64(22 \%)$ & 34 (21\%) & $3(15 \%)$ & $31(22 \% 9$ & $30(23 \%)$ & $5(33 \%)$ & $25(21 \%)$ \\
\hline $\begin{array}{l}\text { Glucosa } \\
\geq 100 \mathrm{mg} / \mathrm{dl}\end{array}$ & 187 (64\%) & $104(65 \%)$ & $14(70 \%)$ & $90(64 \%)$ & $83(63 \%)$ & $11(73 \%)$ & 72 (61\%) \\
\hline $\begin{array}{l}\mathrm{TG} \\
\geq 150 \mathrm{mg} / \mathrm{dl}\end{array}$ & $121(41 \%)$ & $61(38 \%)$ & $7(35 \%)$ & $54(38 \%)$ & $60(45 \%)$ & $6(40 \%)$ & $54(46 \%)$ \\
\hline $\begin{array}{l}\mathrm{HDL} \\
\quad \geq 40 \mathrm{mg} / \mathrm{dl} \text { hombres } \\
\geq 50 \mathrm{mg} / \mathrm{dl} \text { mujeres }\end{array}$ & $240(82 \%)$ & 135 (84\%) & $12(60 \%)$ & $123(87 \%)$ & $105(80 \%)$ & $8(53 \%)$ & 97 (83\%) \\
\hline
\end{tabular}

HDL: lipoproteínas de alta densidad; TG: triglicéridos.

realizar correlación de Pearson entre los niveles de TSH y los componentes del SM no se encontró ninguna correlación significativa.

\section{DISCUSIÓN}

A diferencia de nuestros resultados, en Yemen, India y Turquía encontraron el SM con menos frecuencia que en nuestro país. Entre las prevalencias nacionales de SM en población general de estos países, la de México es la mayor, con un $54 \%$ de acuerdo con los criterios de la IDF ${ }^{16}$. Nosotros, con dos terceras partes de SM en pacientes mexicanos hipotiroideos y prevalencia nacional de la mitad, somos de los países con más alto índice de asociación mundial de estas alteraciones metabólicas y hormonales.

La relación entre hipotiroidismo y SM ha sido estudiada de diferentes maneras, algunas con resultados contradictorios. Chang, et al. encontraron que pacientes con SM tenían más riesgo de padecer hipotiroidismo subclínico, especialmente si tenían presión arterial elevada y niveles altos de triglicéri$\operatorname{dos}^{17}$, mientras que en otro estudio realizado en población portuguesa no encontraron asociación entre los niveles de TSH y la presencia de SM y sus componentes, sin embargo, encontraron que la presencia de anticuerpos antitiroideos tenía una asociación negativa significativa con la presencia del síndrome ${ }^{18}$. Una limitante de nuestro estudio fue que no realizamos pruebas de anticuerpos, por lo que no se puede comparar con estos resultados.

Diversos estudios han encontrado que frecuentemente coexiste obesidad y disfunción tiroidea, donde por un lado la elevación de TSH puede ser una consecuencia de la obesidad y por el otro lado la disfunción tiroidea, especialmente la subclínica, puede ser la causa de la obesidad. Estos pacientes continúan ganado peso y desarrollan dislipidemia. La razón por la cual se relaciona la obesidad con disfunción tiroidea no está del todo clara, solo existen teorías que involucran a la leptina y la autoinmunidad ${ }^{19}$. Esta relación entre obesidad, disfunción tiroidea y dislipidemia podría explicar la alta prevalencia de SM encontrada en nuestro estudio. De igual forma, estos análisis sugieren que un adecuado control del peso podría revertir la disfunción tiroidea y dislipidemia.

En el estudio se encontró con mayor frecuencia el $\mathrm{SM}$ en pacientes con hipotiroidismo subclínico, así 
como mutualidad entre ambas. Se sabe que existe una asociación entre los niveles elevados de TSH y la enfermedad cardiovascular, así como que el SM conlleva mayor riesgo cardiovascular por daño endotelial ${ }^{20,21}$. En un estudio realizado por Razvi, se demostró que regular los niveles de TSH con levotiroxina en pacientes con hipotiroidismo subclínico estaba asociado a la disminución de eventos cardiovasculares $^{22}$.

En conclusión, existe una mayor presencia de SM en pacientes con hipotiroidismo, sobre todo en aquellos con hipotiroidismo subclínico. Se sugiere una búsqueda de ambas enfermedades para un adecuado manejo, enfocando el tratamiento en la pérdida de peso y el control de niveles de TSH, para así tener una disminución de riesgo cardiovascular.

\section{FINANCIAMIENTO}

El Instituto Mexicano del Seguro Social proporcionó recursos de atención médica propios del instituto. No se recibió apoyo económico o beca de ningún fondo de investigación, ni de fuentes externas.

\section{CONFLICTO DE INTERESES}

Los autores declaran no tener conflicto de intereses.

\section{RESPONSABILIDADES ÉTICAS}

Protección de personas y animales. Los autores declaran que para esta investigación no se han realizado experimentos en seres humanos ni en animales.

Confidencialidad de los datos. Los autores declaran que han seguido los protocolos de su centro de trabajo sobre la publicación de datos de pacientes.
Derecho a la privacidad y consentimiento informado. Los autores han obtenido el consentimiento informado de los pacientes y/o sujetos referidos en el artículo.

\section{BIBLIOGRAFÍA}

1. Saklayen MG. The global epidemic of the metabolic syndrome. Curr Hypertens Rep. 2018;20(2):12

2. Eckel RH, Grundy SM, Zimmet PZ. The metabolic syndrome. Lancet 2005;365(9468):1415-28.

3. Alberti KG, Eckel RH, Grundy SM, Zimmet PZ, Cleeman Jl, Donato KA, et al. Harmonizing the metabolic syndrome: a joint interim statement of the International Diabetes Federation Task Force on Epidemiology and Prevention; National Heart, Lung, and Blood Institute; American Heart Association World Heart Federation; International Atherosclerosis Society; and International Association for the Study of Obesity. Circulation. 2009;120(16):1640-5.

4. Eckel RH. The metabolic syndrome. En: Kasper DL, Hauser SL, Jameson JL, Fauci AS, Longo DL, Loscalzo J, editores. Harrison's Principles of Internal Medicine. 19th ed. McGraw-Hill Education; 2015. pp. 2449-54.

5. Roberts CG, Ladenson PW. Hypothyroidism. Lancet. 2004;363(9411):793-803.

6. Surks Ml, Boucai L. Age-and race-based serum thyrotropin reference limits. J Clin Endocrinol Metab. 2010;95(2):496-502.

7. Taylor PN, Albrecht D, Scholz A, Gutierrez-Buey G, Lazarus JH, Dayan CM, et al. Global epidemiology of hyperthyroidism and hypothyroidism. Nat Rev Endocrinol. 2018;14(5):301-16.

8. Udovcic M, Pena RH, Patham B, Tabatabai L, Kansara A. Hypothyroidism and the heart. Methodist Debakey Cardiovasc J. 2017;13(2):55-9.

9. Suh S, Kim DK. Subclinical hypothyroidism and cardiovascular disease. Endocrinol Metab (Seoul). 2015;30(3):246-51.

10. Iwen KA, Schröder E, Brabant G. Thyroid hormones and the metabolic syndrome. Eur Thyroid J. 2013;2(2):83-92.

11. Bonora BM, Fadini GP. Subclinical hypothyroidism and metabolic syndrome: A Common association by chance or a cardiovascular risk driver? Metab Syndr Relat Disord. 2016;14(8):378-80.

12. Yang L, Lv X, Yue F, Wei D, Liu W, Zhang T. Subclinical hypothyroidism and the risk of metabolic syndrome: A meta-analysis of observational studies. Endocrine research. 2016;41(2):158-65.

13. Bamashmos MA, Atef ZA. The prevalence of metabolic syndrome in Yemeni patients with hypothyroidism. MEJFM. 2016;14(6):29-36.

14. Khare J, Nalla S, Wadhwa J, Srivastava P, Reddy B, Deb P. Prevalence of metabolic syndrome in hypothyroidism: Experience in a tertiary center in South India. CHRISMED J Health Res. 2017:4(1):19-22.

15. Takir M, Kizilgul M. Prevalence of metabolic syndrome in hypothyroid patients under Levothyroxine therapy. Medeniyet Medical Journal. 2018.

16. Gutierrez-Solis AL, Datta Banik S, Mendez-Gonzalez RM. Prevalence of metabolic syndrome in Mexico: A systematic review and meta-analysis. Metab Syndr Relat Disord. 2018;16(8):395-405.

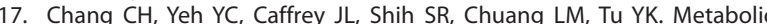
syndrome is associated with an increased incidence of subclinical hypothyroidism - A cohort study. Sci Rep. 2017;7(1):6754.

18. Raposo L, Martins S, Ferreira D, Guimaraes JT, Santos AC. Metabolic syndrome, thyroid function and autoimmunity - The PORMETS Study. Endocr Metab Immune Disord Drug Targets. 2019;19(1):75-83.

19. Gutch M, Rungta S, Kumar S, Agarwal A, Bhattacharya A, Razi SM. Thyroid functions and serum lipid profile in metabolic syndrome. Biomed J. 2017:40(3):147-53.

20. Franca MM, Nogueira CR, Hueb JC, Mendes AL, Padovani CR, Mazeto GM. Higher carotid intima-media thickness in subclinical hypothyroidism associated with the metabolic syndrome. Metab Syndr Relat Disord. 2016;14(8):381-5

21. Inoue K, Ritz B, Brent GA, Ebrahimi R, Rhee CM, Leung AM. Association of subclinical hypothyroidism and cardiovascular disease with mortality. JAMA Netw Open. 2020;3(2):e1920745.

22. Razvi S, Weaver JU, Butler TJ, Pearce SH. Levothyroxine treatment of subclinical hypothyroidism, fatal and nonfatal cardiovascular events, and mortality. Arch Intern Med. 2012;172(10):811-7. 